\title{
Virtual three-phase laboratory exercise during pandemic situation
}

\author{
FELIX A. HIMMELSTOSS ${ }^{1} \&$ KARL EDELMOSER ${ }^{2}$ \\ ${ }^{1}$ Power Electronics Department \\ University of Applied Science Technikum Wien \\ Hoechstaedtplatz 6, 1200 Wien \\ ${ }^{2}$ Institute of Energy Systems and Electrical Drives \\ University of Technology Vienna \\ Gusshausstraße 24-26, 1040 Wien \\ AUSTRIA
}

\begin{abstract}
The Corona pandemic has changed our way to teach, even our laboratory experiments. The exercises for the three-phase systems had to be transferred to the web. The virtual exercises are described and the results are shown. The pros and contras of this way to teach are discussed.
\end{abstract}

Key-Words: - virtual laboratory, three-phase systems, phasor diagram, neutral line interruption, voltage displacement,

Received: June 15, 2021. Revised: October 23, 2021. Accepted: December 9, 2021. Published: January 3, 2022.

\section{Introduction}

For many years the first author has taught a course for Industrial Electronics in the second year of the Bachelor course for Electronics. Only one week is assigned for the chapter three-phase systems (references are e.g. [1, 2]), one lecture for the basics and one lecture for some calculation examples. A laboratory exercise rounds off this theme. In this laboratory exercise the students have to wire a threephase load connected in star with a $400 \mathrm{~V}$ mains (for security reasons, a one to one isolation transformer is used). In the circuits the voltages across the load and the currents through the wires are measured. First we use a symmetrical resistive load, then some asymmetry is included and several errors are induced (disconnection of wires). The ammeters and the voltmeters are read for the experiments and phasor diagrams are constructed. This is very helpful to get a better understanding of this topic. During the pandemic situation in the study year 2020/21 all lectures were transferred to Zoom lectures. Therefore, an online exercise which simulates the practical laboratory exercise was made. The useful simulation program LT-Spice [3] was used, which can be loaded down with no charge.

\section{Basic experiments}

\subsection{Experiment 0: Power flow}

The starting point is the question: why do we use a three-phase system? It is easy to understand that with more wires more energy can be transferred and the other point (which was already discussed in the lecture) is the fact that the power transfer is nearly constant compared to a pulsating one in a two wire system. This was demonstrated at first (Fig. 1). The voltage sources with the value zero represent the ammeters A1, A2, A3, A4. The voltages across the load resistors are equal to the phase voltages (this is also in reality, because the voltage drop across the ammeters can be neglected; in reality we use seven measurement devices three voltmeters and four ammeters). Fig. 2 shows the constant power flow and the three pulsating ones for the three one-phase systems. Also depicted are the currents through all four wires and the phase voltages.

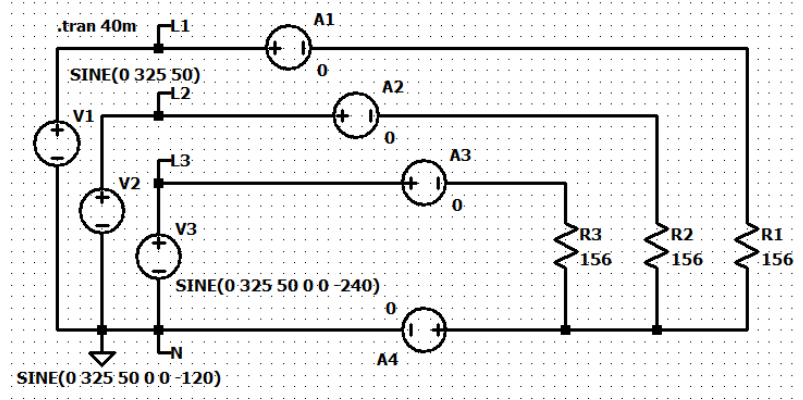

Fig. 1. Symmetrical load

Contrary to a single phase system which has a pulsating power flow, the power flow in a symmetric three-phase system is constant. So no mechanical stress at the generator is caused. In a one-phase system the constant mechanical power from the turbine and the pulsating electrical power at the electrical output lead to a large mechanical stress with a frequency of two times the frequency of the mains . 


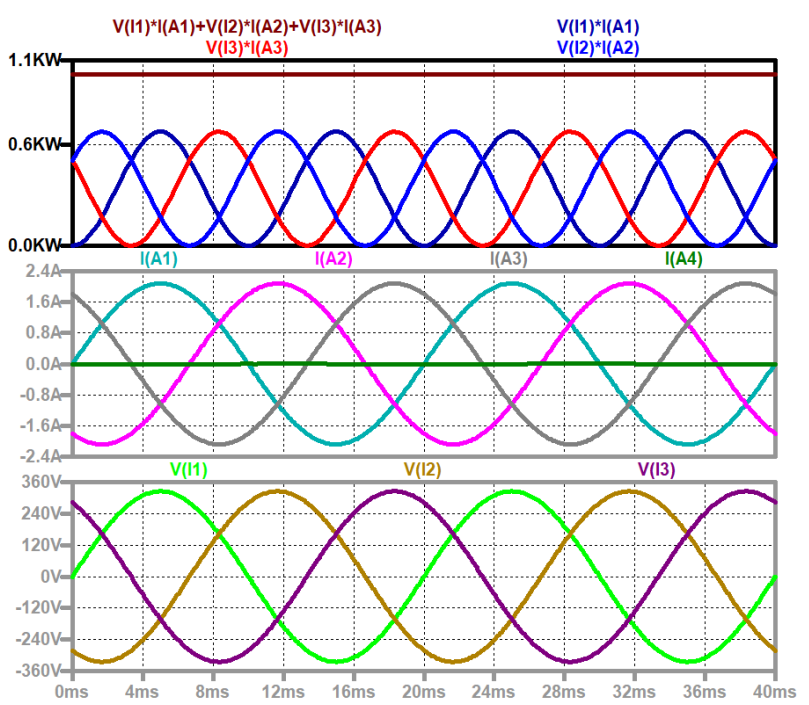

Fig. 2. Up to down: power flow of the complete three-phase system, power flow of the three phases; currents through the lines; phase voltages

\subsection{Experiment 1: Symmetrical load}

The model (Fig. 1) is equal to the setup in the laboratory. The symmetrical load consists of resistors and the currents are measured in each line. The voltage sources with the value zero represent the current measurement devices (ammeters). To get the rms-value as in reality (keep in mind that in the laboratory we only get the rms-values with the measurement devices), one must display one or an integer manifold of periods and use the key $<$ control>, and the left key of the mouse when pointing with the cursor at the symbol of the desired signal in the probe window. The signals (phase currents and phase voltages) are shown in Fig. 2 in the two lower pictures. The rms-values are 1,473 A in the phases and zero in the neutral line. The current in the neutral is the sum of the phase currents. The phasor diagram (Fig. 3) shows that the current in the neutral is zero.

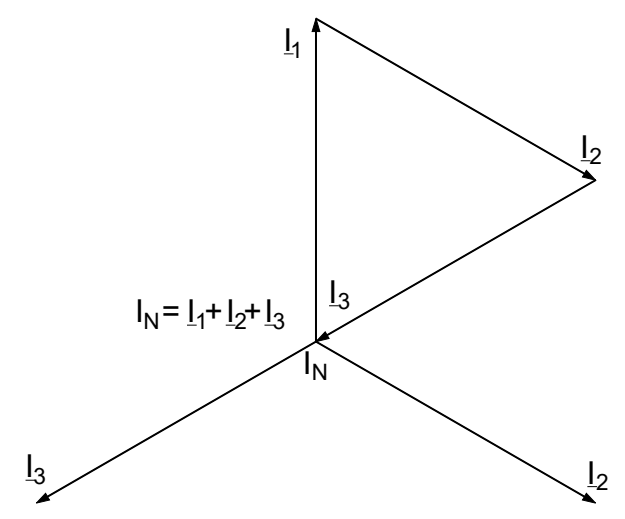

Fig. 3. Phasor diagram for experiment 1

\subsection{Experiment 2: Unsymmetrical load}

The load resistor in phase one is reduced to the half of the original value (Fig. 4). Now the current in phase one is doubled. The load is unsymmetrical and the current is equalized by a current through the neutral line (Fig. 5).

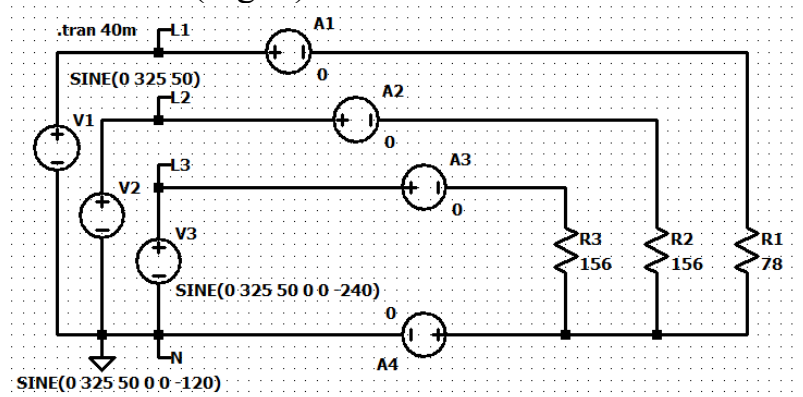

Fig. 4. Experiment 2: unsymmetrical load

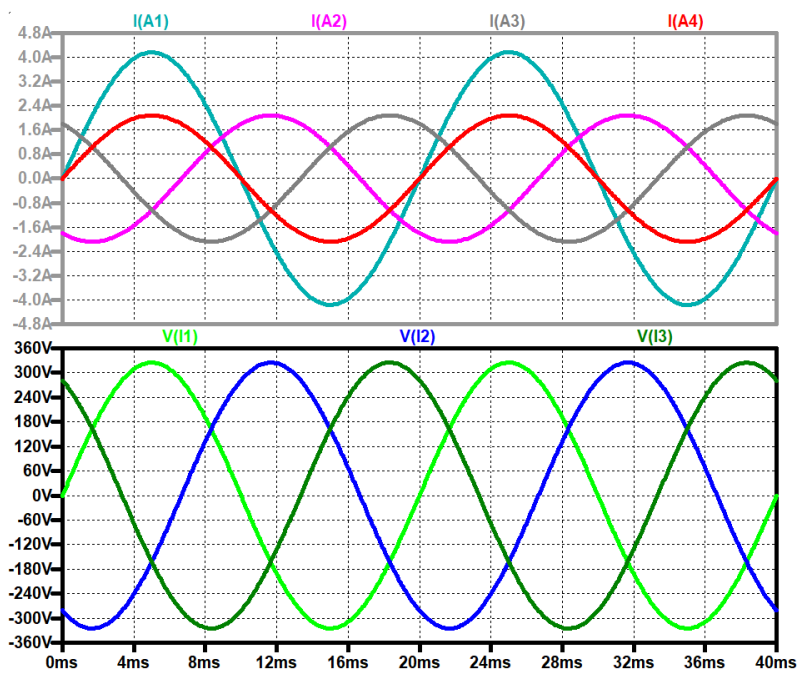

Fig. 5. Experiment 2 up to down: currents through the wires; phase voltages

Reading from the "ammeters" results in $\mathrm{I} 1=2.945 \mathrm{~A}$, $\mathrm{I} 2=\mathrm{I} 3=\mathrm{I} 4=1.473 \mathrm{~A}$. This leads to the phasor diagram Fig. 6.

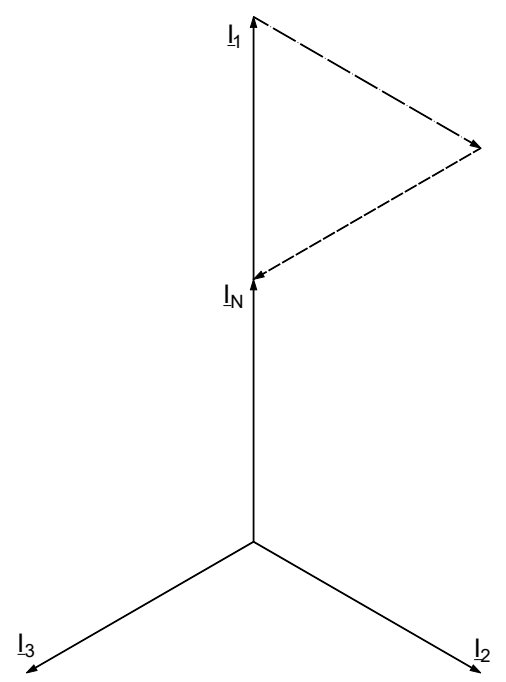

Fig. 6. Phasor diagram for experiment 2 


\subsection{Experiment 3: interruption of phase 1}

Now we interrupt phase one (Fig.7). This could be by the blow of a fuse. Now we have only two working phases and again a current has to flow through the neutral line.

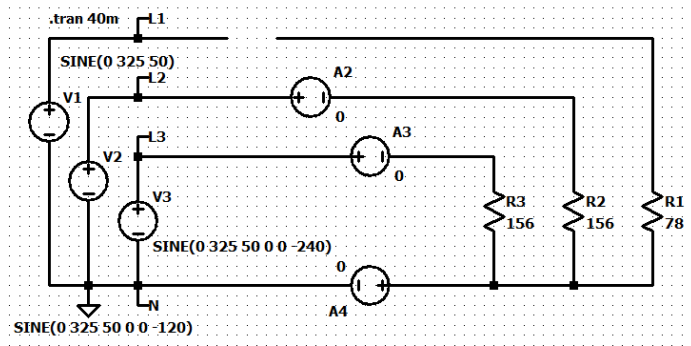

Fig. 7. Experiment 3: interruption of phase 1

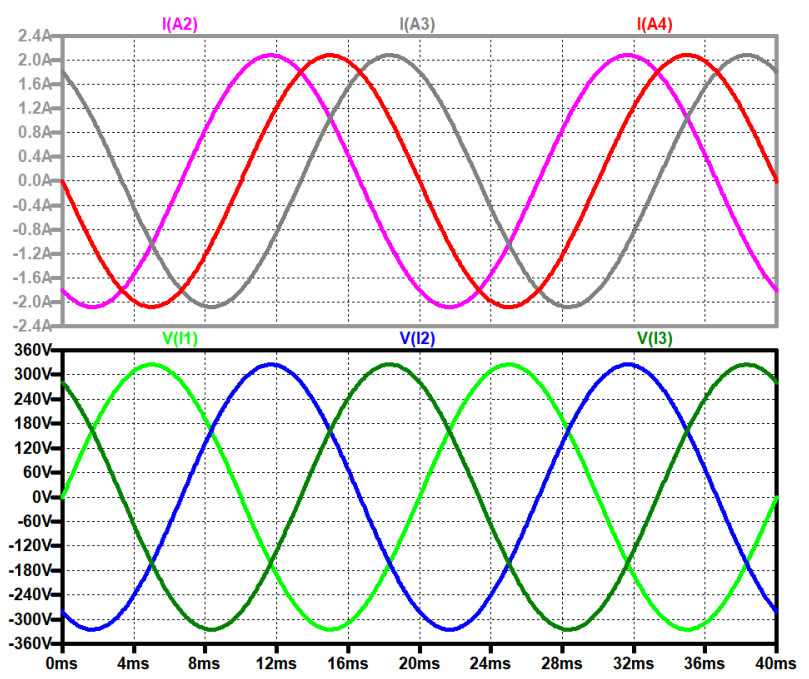

Fig. 8. Experiment 3 up to down: currents through the wires; phase voltages

Reading our "ammeters" leads to zero for phase one and again $1.473 \mathrm{~A}$ for all other currents. To equalize the system, again a current has to flow through the neutral line. Constructing the phase diagram (Fig. 9) helps again to understand this.

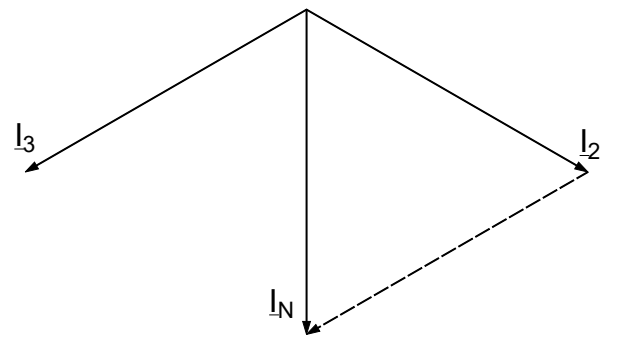

Fig. 9. Phasor diagram for experiment 3

\subsection{Experiment 4: Additional interruption of the neutral line}

Now a second error occurs, the neutral line is also interrupted (Fig. 10).

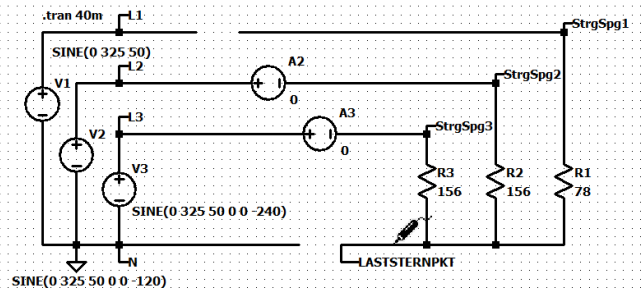

Fig. 10. Experiment 4: interruption of phase 1 and of the neutral

Now two things occur: the star point of the source which is normally grounded and the star point of the load are different and a dangerous voltage between the star point of the load and earth can occur, and secondly the voltages across the load differ from the phase voltages. Fig. 11 shows the shift of the two star points, the voltages across the loads, the phase currents, and the phase voltages. Reading our "ammeters" shows the same current 1.276 A in line 2 and line 3 . The voltages across R 2 and R 3 are the same.

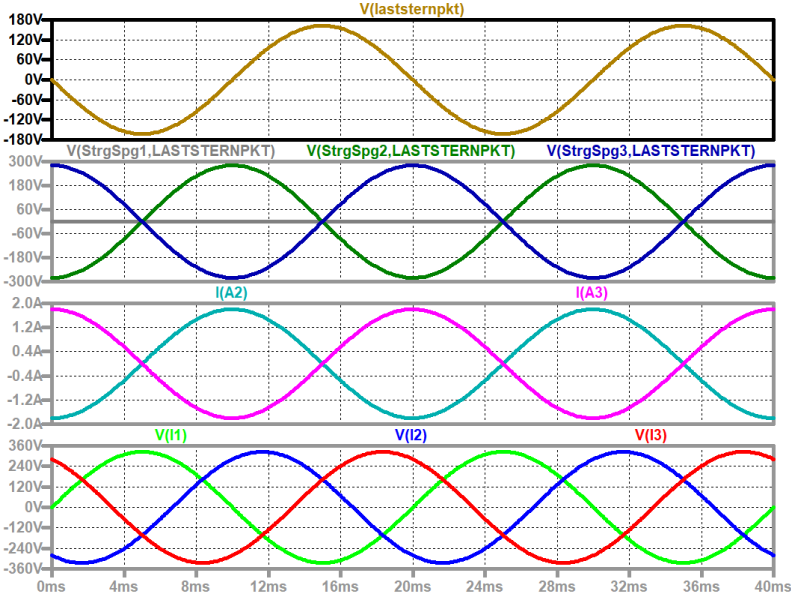

Fig. 11. Experiment 4 up to down: shift of the star point, voltages across the loads, phase currents, phase voltages

The phase current is the same in both phases. Across the series connection of both load resistors lies now the line-to-line voltage. The voltage across the two load resistors is now only $200 \mathrm{~V}$ (exact $199 \mathrm{~V}$ in our case). The most interesting fact is the voltage difference between the earthed voltage source and the star point of the load. There is a voltage shift of $115 \mathrm{~V}$, already a dangerous value! The interruption of the neutral line can be very dangerous when the load is unsymmetrical!

The construction of the phasor diagram (Fig. 12) helps for better understanding. The construction follows Kirchhoff's voltage law. The phase voltage is the sum of the voltages across the load and the difference between the two star points. One gets 


$$
\begin{aligned}
& \underline{V}_{2}=\underline{V}_{\text {StrSpg2 }}+\underline{V}_{\text {LASTSTERNPKT }} \\
& \underline{V}_{3}=\underline{V}_{\text {StrSpg3 } 3}+\underline{V}_{\text {LASTSTERNPKT }} .
\end{aligned}
$$

With the measured values and with the help of a pair of compasses one can construct the phasor diagram (Fig. 12).

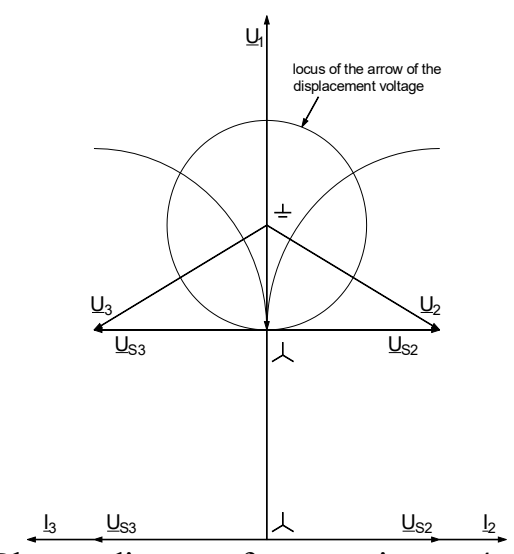

Fig. 12. Phasor diagram for experiment 4

\subsection{Experiment 5: repair of the system}

The system is again the system as shown in Fig. 1. When the load is equal for all three phases, an interruption of the neutral point does not matter. (High voltage transmission lines have only a thin earth line which conducts only the current caused by some unavoidable asymmetry and are necessary in case of a system fault; district lines in the tens of $\mathrm{kV}$ range have only three lines.)

There is no voltage difference between earth (the neutral point or star point of the source) and the star point of the load.

\subsection{Experiment 6: Capacitive load in phase 1}

The system is now repaired, but we change the load of phase 1 by a capacitor which draws about the same current as the original resistor (Fig. 13). From the reactance of a capacitor

$$
X_{C}=\frac{1}{\omega C} \text {. }
$$

one gets

$$
C=\frac{1}{\omega X_{C}}=\frac{1}{2 \pi f \cdot 156}=20.4 \mu \mathrm{F} .
$$

In our laboratory we have $20 \mu \mathrm{F}$ capacitors which can be used in this case. Now the load is asymmetric and a current is flowing through the neutral line. The results are shown in Fig. 14 and the phasor diagram in Fig. 15. The "ammeters" show $\mathrm{I} 1=1.443 \mathrm{~A}, \mathrm{I} 2=1.473 \mathrm{~A}, \mathrm{I} 3=1.473 \mathrm{~A}, \mathrm{I} 4=2.063 \mathrm{~A}$ (measured during the fourth and fifth period).

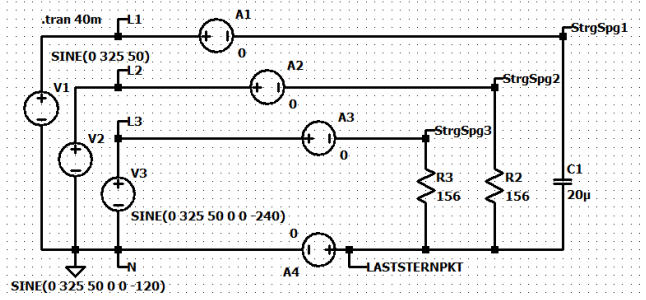

Fig. 13. Experiment 6: capacitive load in phase 1

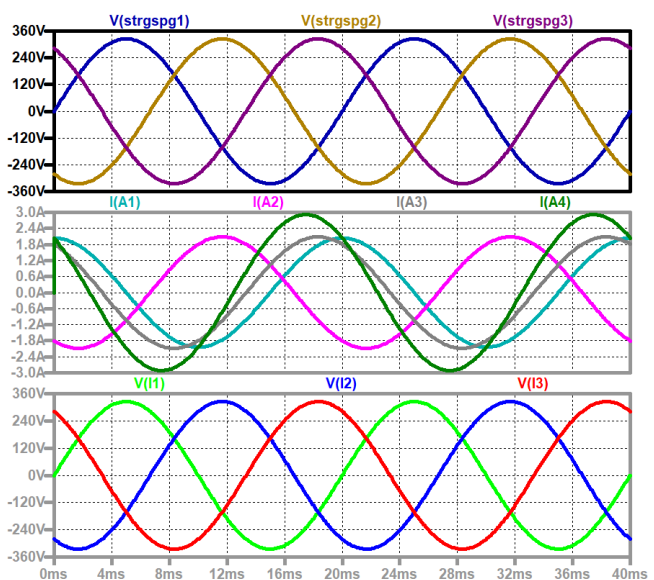

Fig. 14. Experiment 6 up to down: voltages across the load, current through the lines, phase voltages

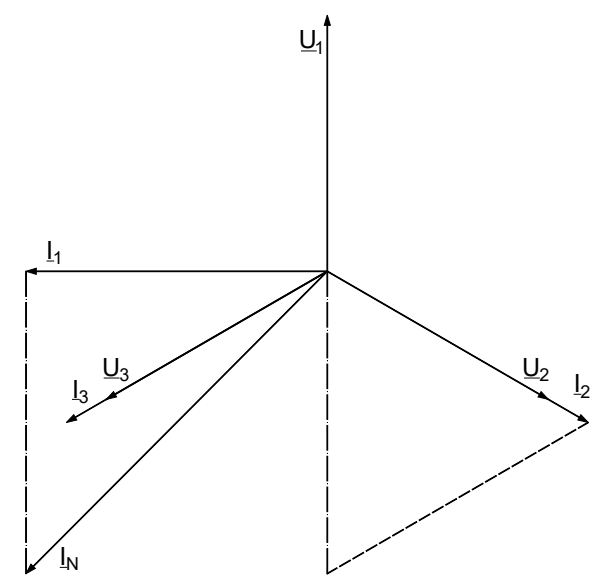

Fig. 15. Phasor diagram for experiment 6

2.8 Experiment 7: capacitive load in phase 1 and interruption of the neutral line What happens when the neutral line is now interrupted (Fig. 16)?

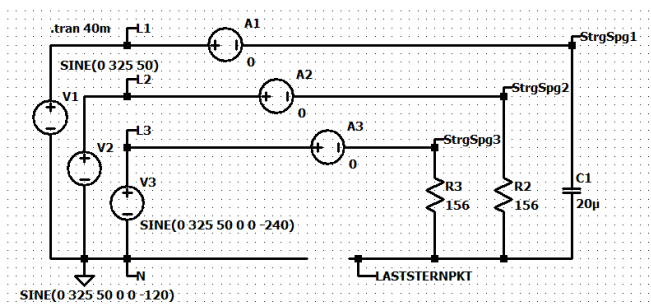

Fig. 16. Experiment 7: interruption of the neutral line 
For the measurement one has to check that the system is in the steady state. Fig. 17 shows the shift of the load star point, the voltages across the load, the current through the phases, and the phase voltages.

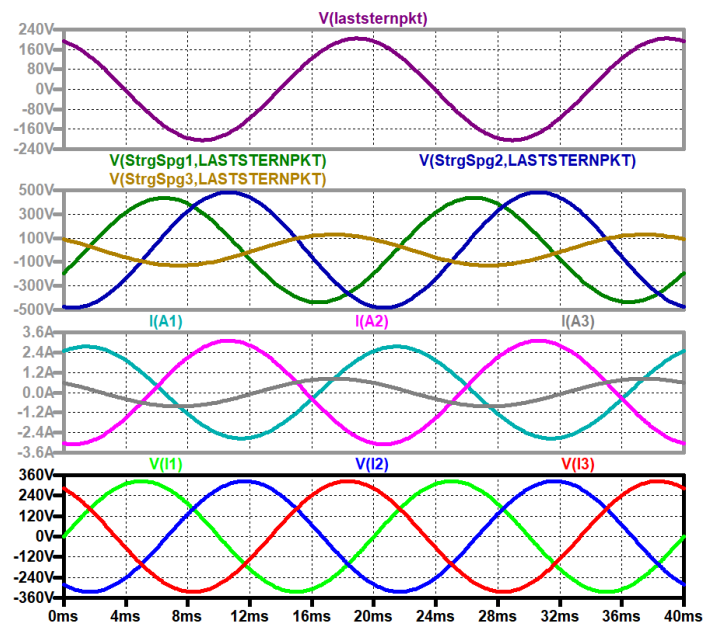

Fig. 17. Experiment 7 up to down: shift of the load star point, voltages across the load, current through the phases, phase voltages

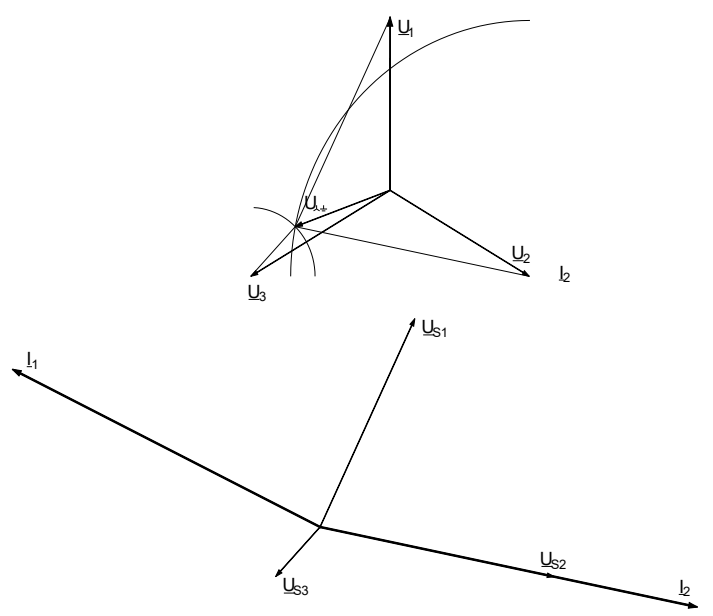

Fig. 18. Phasor diagram for experiment 7

For the steady state one gets the values $\mathrm{I} 1=1.9443 \mathrm{~A}, \quad \mathrm{I} 2=2.1905 \mathrm{~A}, \mathrm{I} 3=0.5873 \mathrm{~A}$ for the currents, for the load voltages $\mathrm{UC} 1=309,5 \mathrm{~V}$, $\mathrm{UR} 2=341.8 \mathrm{~V}$, UR3 $=91.68 \mathrm{~V}$, and for the displacement of the star point of the load $144.44 \mathrm{~V}$ (the measurement was done in the fifth period). The voltage across load R2 is much higher than the normal phase voltage of $230 \mathrm{~V}$. Therefore, this load can be destroyed (e.g. a TV set or other electronic equipment)! The load across R1 is much lower than the normal voltage and this can lead to a malfunction. The voltage across the capacitor is also significantly higher than the nominal voltage. Asymmetric load and interruption of the neutral can be very dangerous. Again the drawing of the phasor diagram (Fig. 18) enhances the understanding. The current in phase one leads the voltage across the load capacitor by ninety degrees.

\subsection{Experiment 7: star point displace- ment Sternpunktverschleppung}

The last of our basic experiments shows that it can be very dangerous when the neutral is interrupted. For example, two flats are supplied from the same source. Therefore, they use the same neutral line. If this line is interrupted and the load in one flat is asymmetric, then not only the voltage at the loads can destroy the loads in this flat, but also in the neighboring one!

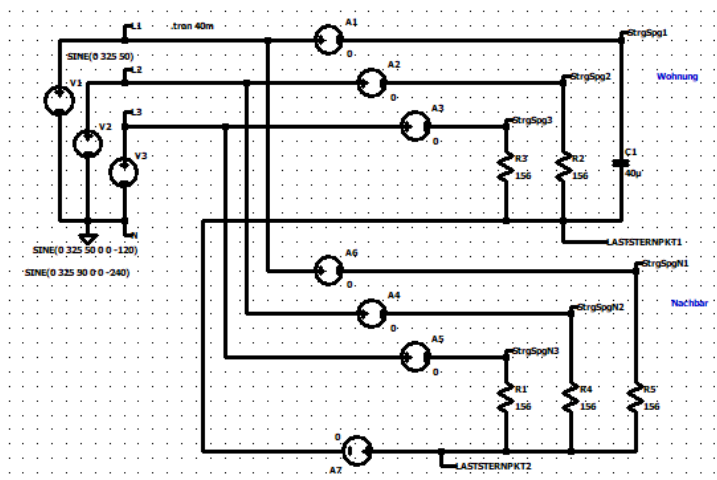

Fig. 19. Experiment 8: Voltage displacement

\section{Extensions of these laboratory experiments}

Depending on the time one can expand these experiments, e.g. the load can be connected in triangle, the three-phase system can be rectified, or the load can be an induction machine.

\subsection{Experiment 9: Triangle load}

Fig. 20 shows the circuit diagram for a triangle load. The current in all load branches is also measured. One can extend this experiment by studying the influence of interruptions of different wires after first considering it. First it must be done with paper, pencil, and India rubber!

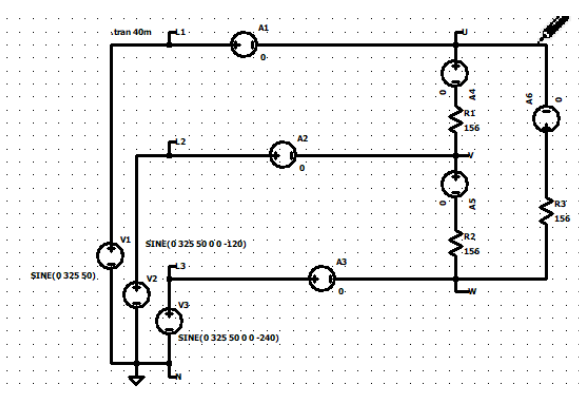

Fig. 20. Experiment 9: triangle load 


\subsection{Experiment 10: three-phase rectification}

Another expansion could be a rectification [4] with a B6 bridge (Fig. 21). This should, however, also be studied with paper and pencil before. The results are shown in Fig. 22, where the current through the lines and the phase voltages are depicted together. In the last picture the rectified output voltage and the input voltages are shown. The currents through the diodes and the voltages across them can be studied in another experiment. In a next step the influence of inductors in the lines and in the load can be treated.

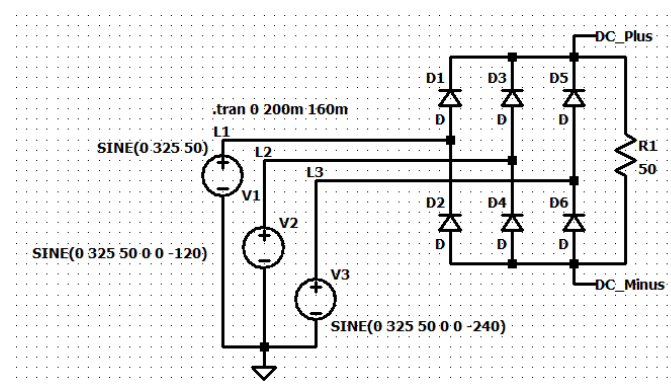

Fig. 21. Experiment 10: B6 rectifier

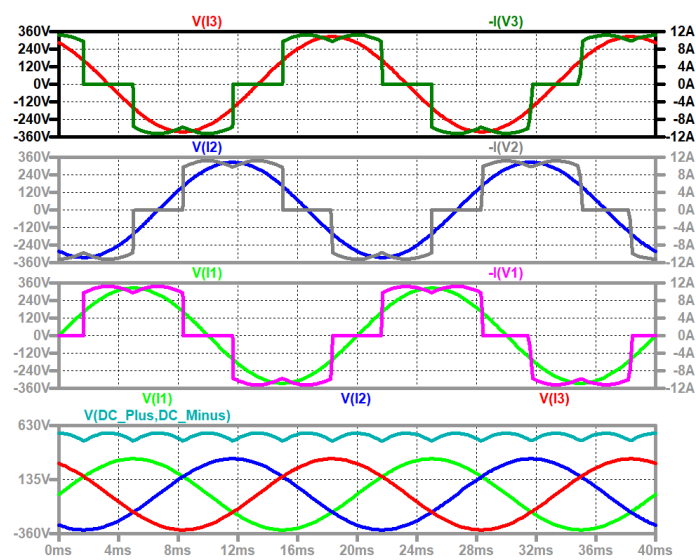

Fig. 22. Experiment 10 up to down: voltage and current phase 3 , voltage and current phase 2 , voltage and current phase 1 , rectified voltage and phase voltages

\subsection{Experiment 11: induction machine}

In this experiment an induction machine modelled by the T-model can be treated. Fig. 23 shows a machine in the nominal point.

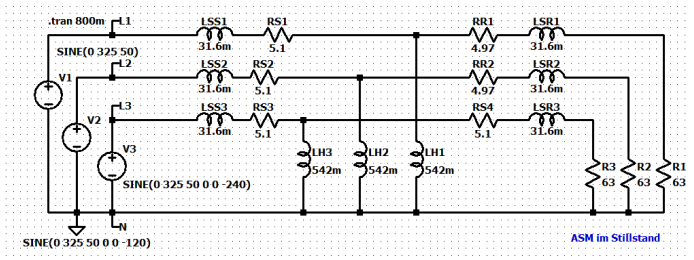

Fig. 23. Experiment 11: induction machine at the nominal point

\section{Conclusion}

The main idea of these exercises in the laboratory is that the students of the Electronics curricular should at least work one time in their course at the $400 \mathrm{~V}$ three-phase mains. To reduce the risk of hazards an isolation transformer separates the circuits from the distribution network. We also use cables with connectors which are especially isolated. But carelessness can always occur, e.g. if the voltages should be measured and the measurement device is used as an ammeter or a cable is wrongly connected. In the virtual lab exercise these errors have no dangerous consequences and there is no stress for the responsible lecturer (I was always glad when these exercises were finished). The second task of these experiments is to practice measurements and to construct the phasor diagrams. Measurements in the virtual exercises are easier, because there are no fluctuations of the voltages. The instruments have to be read at the same time (the students now make a picture with their mobile phones to get the results at the same moment). The problems with the construction of the phasor diagrams are the same both after the real measurement or after the simulation (when all ammeters and voltmeters are working correct). The results of the simulation and their interpretation are the same and help to get a better understanding of the three-phase system. The exercises last shorter than in reality and in the same available time more experiments can be carried out. Interested students can repeat these experiments at home and change the tasks and check other problems. Nevertheless, the practical work with hardware should be done in our courses.

\section{References:}

[1] Olle I. Elgerd, and Patrick D. van der Puije, Electronic Power Engineering, Chapman \& Hall, 1998.

[2] Mohamed E. El-Hawary, Electric Power Systems, IEEE Press, 1995.

[3] Analog Devices, LTspice (can be downloaded for free)

[4] Ned Mohan, Torr M. Undeland, William P. Robbins, Power Electronics, John Wiley \& Sons, 2003.

\section{Creative Commons Attribution License 4.0 (Attribution 4.0 International, CC BY 4.0)}

This article is published under the terms of the Creative Commons Attribution License 4.0

https://creativecommons.org/licenses/by/4.0/deed.en_US 